Doniazade de May Telmissany et Un tout petit rien de Camille Anseaume

\title{
Doniazade de May Telmissany et $U n$ tout petit rien de Camille Anseaume, étude psychocritique
}

\author{
Ayman Amin ELGHANDOUR \\ Professeur adjoint à la Faculté de Pédagogie de Tanta
}

\begin{abstract}
"Dans une société où l'individu appartient d'abord au clan ou à la famille, la femme plus encore que l'homme, n'a pas d'existence sociale. Écrire son autobiographie, c'est s'en donner une malgré l'opposition ambiante. Dévoiler sa vie devient un acte révolutionnaire." (Tadié, 1996, p.79)
\end{abstract}

La présente étude vise à mettre l'accent sur la relation entre la création et la procréation à travers Doniazde de May Telmissany et $U n$ tout petit rien de Camille Anseaume. Notre choix remonte à la nature de ces deux romans féminins qui présentent l'expérience de la grossesse et de l'accouchement. Nous y trouvons deux héroïnes enceintes à la recherche de leur identité, ainsi que le mélange de l'écriture et de la maternité.

Notre choix des deux romancières contemporaines donne une valeur à cette étude dont l'intérêt réside dans sa double nature, d'une part, elle contribue au domaine riche de la littérature comparée qui se distingue "par la recherche de liens d'analogie, de parenté et d'influence" (Pichois et Rousseau, 1967, p.174) et qui nous aide à découvrir l'autre et "à prendre conscience des problèmes de communication et de compréhension qu'impliquent confrontation et comparaison;" (Souiller, 1997, p. XVI) d'autre part, les deux ouvrages de notre corpus sont des récits de vie, appartenant au genre romanesque qui "se caractérise par une ambition panoramique, par sa faculté à mettre en forme la totalité de l'expérience humaine." (Boyer, 1996, p. 127) A la différence de la poésie qui excite au rêve et de l'essai qui suscite la réflexion, "le roman, lui, raconte, décrit, fait parler ou discourt." (Valette, 1993, p. 19) 
Comme les deux romancières essayent à travers Doniazade et Un tout petit rien, de raconter ce qui les soulage et leur sert de thérapie, nous adoptons la psychocritique qui "n'étudie pas l'œuvre totale mais sa base inconsciente, c'est-à-dire le mythe personnel. Interpréter le mythe n'est pas expliquer l'œuvre entière." (Mauron, 1996, p. 312) Loin d'étudier le génie du créateur, cette méthode vise à dévoiler son inconscient et les motifs qui l'ont poussé à écrire. Cette méthode compte sur quatre opérations: la superposition de divers textes d'un même auteur pour dégager les éléments répétés; le réseau obsédant ou les métaphores obsédantes qui relèvent les images et les mots privilégiés de cet auteur; le mythe personnel qui s'avère l'élément le plus important et qui nous aide à bien saisir la personnalité du créateur; et enfin la biographie de l'auteur afin de vérifier ce qu'il a présenté dans le texte.

En fait, la psychanalyse et la littérature sont étroitement liées. La première ne se contente pas de contribuer à la performance de l'autre, à révéler ses secrets, mais elle en reçoit ce qui l'aide à s'enrichir et purifier ses concepts. Noël (1996, p. 48) affirme que "La relation entre la psychanalyse et la littérature est à double sens: chacune emprunte et prête à l'autre, toutes les deux trouvent un bénéfice dans cet échange."

Nous essaierons à travers cette étude de mettre en évidence les inquiétudes et les préoccupations de ces deux écrivaines enceintes, ainsi que leurs fantasmes reliés au fœtus et à la grossesse. Nous allons examiner les deux romans de notre corpus afin d'en dégager ce que ces deux romancières avaient de commun. Nous tenterons de répondre à ces questions: Qui sont ces deux auteures? Pourquoi ont-elles écrit Doniazade et Un tout petit rien? Ya-t-il des aspects similaires et différents pendant leur grossesse? Celle-ci a-t-elle influencé leurs écrits? Ont-elles présenté une autobiographie ou une autofiction? Quelles sont leurs participations à l'éveil de leur société?

Comme la méthode psychocritique s'intéresse à étudier divers textes du même auteur, comparant les résultats acquis à sa vie 
Doniazade de May Telmissany et Un tout petit rien de Camille Anseaume

personnelle, afin de "saisir, dans l'œuvre de chaque écrivain, les manifestations d'un moi profond," (Mauron, 1996, p. 14) nous devrons mettre en lumière la vie et la production littéraire de nos deux romancières égyptienne et française avant d'étudier en détails les deux romans de notre corpus. Nous sommes éclairé par les dires de Charles Mauron: "L'ouvre ne peut que retentir sur la vie, à qui elle prend une grande part d'énergie et à qui elle impose des exigences très strictes." (p. 331)

\section{La biographie des deux auteures et la superposition de leurs textes}

May Telmissany est née au Caire en 1965, dans le quartier d'Héliopolis. Elle est à la fois nouvelliste, romancière, scénariste, traductrice et critique de cinéma, influencée par son père le réalisateur Abdel Kader Telmissany (1924-2003). Ce dernier a étudié le cinéma à Paris et est devenu un de ses pionniers en Égypte. Hafid Adnani (2014) nous informe que les El-Telmissany "sont originaires de Tlemcen en Algérie."

Après avoir obtenu sa licence ès lettres françaises, May Telmissany a commencé sa carrière professionnelle comme assistante à la Faculté des Lettres de Menoufiya (1988-1992), puis à l'Académie des Arts au Caire jusqu'en 1995 où l'Université du Caire lui a attribué une maîtrise ès lettres françaises. Pendant cette période, elle était aussi présentatrice de programmes français à la Radio Le Caire. A la fin des années 1990, elle est débarquée au Canada où elle a achevé son doctorat ayant pour titre La hara dans le cinéma égyptien. Elle est maintenant professeure de littérature comparée à la Faculté des Études supérieures et postdoctorales, à l'Université de Montréal.

L'œuvre de May Telmissany se distingue par sa variété. Elle a écrit trois recueils de nouvelles: Gravures répétées (1995), Trahisons mentales (1998), Eil magique (2017) et trois romans: Doniazade (1997), Héliopolis (2001), A capella (2014) sans oublier ses Murailles $d u$ Paradis (2010), écrites à la manière des mémoires. Elle a de même écrit les scénarios de Double $V$, vidéo réalisée dans le cadre des 
Ayman Amin ELGHANDOUR

ateliers cinématographiques à l'Université de Montréal en 2001, d'Alexandrie jour et nuit, documentaire réalisé par son père et projeté au festival Les Alexandries du monde en 1994. Aussi a-t-elle écrit des articles académiques dont on peut citer Latifa el Zayat, La presse cinématographique, 25 films documentaires lauréats de prix internationaux, y compris Les derniers bains du Caire, ce livre qu'elle a rédigé en collaboration avec Ève Gandossi. Elle a traduit en arabe Le livre ailé, Momo et Loulou de Mona Latif-Ghattas ${ }^{1}$, Les grandes écoles esthétiques, Pourquoi lire les classiques, lire le théâtre, Trois pièces de Fernando Arrabal et Les cinémas arabes. Inutile de multiplier toute son œuvre, il convient de souligner ses romans.

Commençons tout d'abord par Doniazade, son premier roman et l'un des deux volets de notre corpus. Cet ouvrage "a reçu le prix Arte Mare (Bastia, France) et le prix d'encouragement de l'État (Le Caire, Égypte) pour le meilleur roman autobiographique." (Wikipedia, May_Telmissany, "S.D.") Publié en arabe par Sharqiyât en 1997, il a été traduit en allemand, anglais, espagnol et néerlandais. Mais ce qui nous importe, c'est la version française, parue aux Actes Sud en 2000.

Dans son premier roman, May Telmissany nous décrit d'une manière détaillée une souffrance appartenant uniquement au monde féminin. La narratrice a perdu sa fille qui n'avait vécu qu'un seul jour. La romancière y exprime ses peines et ses douleurs personnelles. C'est ce qui rend son texte plus sincère et plus authentique. Cette sincérité est justifiée par May elle-même, qui nous confie dans un entretien avec Saïda Shérif: "le roman a été écrit immédiatement après l'incident et je l'ai mis dans le tiroir presque deux ans avant que j'ose le publier."2 (Shérif, 2003)

Avec Héliopolis, son deuxième roman, May nous présente une autofiction, "revisitant les demeures successives par lesquelles elle et ses proches sont passés." (Hassan, 2016, p. 125) C'est l'histoire de

1 - Mona Latif Ghattas est une poétesse égyptienne, née au Caire en 1946. C'est elle qui a traduit les deux premiers romans de May Telmissany.

$$
\text { 2ـ "كتبت الرو اية بعد الحادث مباثرة, فوضعتها في الدرج لمدة عامين قبل أن أجر أ على نشر ها" }
$$


Doniazade de May Telmissany et Un tout petit rien de Camille Anseaume

"Micky, Mahy de son vrai nom." (Telmissany, 2001/2003, p. 11) Adulte, celle-ci nous fait voir de près Héliopolis, sa ville natale. Nous la voyons entourée par quatre femmes; il s'agit de la mère, la grandmère et ses deux tantes. Ces dernières contribuent à renforcer l'existence féminine dans le roman et l'enrichissent grâce à leurs détails quotidiens.

A capella, son troisième roman, est premièrement publié en feuilleton dans Rosalyousef, un journal égyptien, adapté plus tard au cinéma en 2016 par le réalisateur Tamer Sami dans son film Pomme d'Ėve. Ce roman raconte l'histoire de deux amies: Mahy est une femme traditionnelle; mariée, elle mène une vie aisée; l'autre s'appelle Aïda et représente le contraste. Divorcée, elle assume la responsabilité de son enfant, menant une vie agitée et tourmentée. May nous semble prudente de ne pas déterminer le cadre spatio-temporel afin d'inscrire son roman dans une forme d'universalité. Cependant elle nous laisse quelques signes désignant que l'action se déroule au Caire moderne. Nous en citons le fleuve, les coutumes sociales et les prénoms Adel, Aïda, Karim.

Quant à Camille Anseaume, j'avais du mal à trouver des informations propres à sa vie personnelle. Ceci revient au manque des références biographiques. Aucune notion sur sa naissance, seulement rares sont les informations sur ses origines rouennaises. Elle est célèbre par son blog Café des filles, devenu plus tard Une odeur de café. Grâce à ce blog, Camille a pu tisser des liens avec ses lecteurs, leur racontant ses joies et ses peines. Ce blog a été "élu coup de cour de la rédaction de Elle en son temps." (Isabelle, 2014) Elle s'est consacrée à la presse féminine et aux écrits pratiques. "Sous le pseudonyme de Marie Thuillier, elle est aussi l'auteur des livres humoristiques comme le Guide de survie de la jeune maman (2014) et Perles de Parents (2015)." (Babelio. "S.D.") Bien qu'elle ne commence sa carrière romanesque qu'en 2014, elle a pu présenter à ses lecteurs trois romans successifs. Nous allons sans retarder les présenter. 
Ayman Amin ELGHANDOUR

Publié en 2014 aux Éditions Kero, Un tout petit rien, le deuxième volet de notre étude, raconte l'histoire de Camille, le personnage principal. Celle-ci tombe enceinte d'un homme qui ne veut pas qu'elle garde le fœtus. L'héroïne reste quelques semaines, hésitée et oscillée entre la grossesse et l'avortement. Personne ne l'influence, ni ses amies ni sa famille. Elle décide enfin de continuer son parcours vers la maternité, même si elle devient mère célibataire. De plus, elle réussit à imposer son choix à tous ceux qui l'entourent.

Deux ans après son premier roman, Camille Anseaume nous présente Ta façon d'être au monde où elle reprend sa plongée dans l'univers féminin. A travers cette fiction grave, elle met l'accent sur deux amies d'enfance tout à fait différentes; l'une s'avère timide, inquiète alors que l'autre est courageuse et souriante. Nous les voyons ensemble à Paris, à la recherche de l'indépendance et des premiers amours. Tant le temps passe, tant leur amitié croit. Mais un événement tragique les sépare; il s'agit de la mort qui met fin à toute chose, même à la jeunesse.

L'année 2018 témoigne de la parution de Quatre murs et un toit, son troisième roman. Cet ouvrage autobiographique est consacré à la maison de son enfance que son père a décidé de vendre. L'héroïne va à Rouen et s'y installe en une semaine. Elle s'imprègne de la maison qui évoque sa nostalgie et la replonge dans ses souvenirs avec son frère et ses sœurs. Elle trouve dans chaque coin les souvenirs heureux et malheureux de sa vie d'antan. Comme Camille écrit ce roman pour faire ses adieux à cette maison, elle consacre un chapitre à chaque endroit: le banc de l'entrée, la salle à manger, la chambre parentale, la salle de bains et le cellier.

Nous avons remarqué que les écrits de May Telmissany et Camille Anseaume se ressemblent, malgré leur appartenance à deux générations et deux civilisations différentes. Chacune d'entre elles a présenté trois romans basés sur des thèmes féminins. De plus, leur création romanesque est composée de deux autobiographies et d'une fiction. Par hasard leur premier roman est consacré à révéler leur expérience pendant la grossesse. A travers leur autre ouvrage 
autobiographique, les deux romancières ont évoqué leur nostalgie au passé, mettant l'accent sur leur milieu et leurs souvenirs; l'une a présenté Héliopolis, son quartier natal, et l'autre a décrit d'une manière détaillée sa maison d'enfance à Rouen. Même le thème de leur roman fictif est identique. Elles nous brossent le portrait des deux amies opposées dont l'une libérée et l'autre soumise aux traditions.

\section{Les métaphores obsédantes dans les deux ouvrages de notre corpus}

Les métaphores obsédantes sont les mots et les expressions qu'un écrivain favorise. Répétées, elles constituent un réseau significatif, exprimant l'inconscient de ce dernier. En les dégageant, nous remarquons que les métaphores obsédantes dans les deux ouvrages de notre corpus, concernent des thèmes variés: le défi, la féminité et la nostalgie du passé, reprise plus tard dans Héliopolis et Un toit et quatre murs; mais les métaphores les plus évidentes sont la mort, la tristesse dans Doniazade, la grossesse et l'humour dans Un tout petit rien.

Le texte de May fourmille d'expressions tristes reflétant l'état de l'héroïne de Doniazade qui a perdu son enfant et que nous voyons livrée à ses deuils personnels. Bien qu'elle possède un autre enfant qui s'appelle Chihabeddine, cette perte l'a poussée à douter de sa féminité et de sa maternité; elle semble obsédée par la mort, présente tout au long du roman. Regardons le tableau suivant:

\begin{tabular}{|l|l|}
\hline \multicolumn{1}{|c|}{ Métaphores obsédantes dans Doniazade } & Le thème \\
\hline Tant de mots de consolation (p.9) & La tristesse \\
Je pleure à haute voix (p. 10) & \\
Triste comme un puits tari (p. 10) & \\
La tristesse est un fil qui relie la bouche au cour (p. 22) & \\
\hline Dans son petit linceul (p.9) & La mort \\
L'acte de décès (p. 11) & \\
Elle est sauvée et l'enfant? Morte. (p.12) & \\
La morgue de l'hôpital (p. 14) & \\
Elle est sortie de ma tombe vers la sienne. (p.20) & \\
\hline
\end{tabular}


De son côté, Anseaume met l'accent sur la narratrice enceinte dont la grossesse est une étape bouleversante dans sa vie. Fusionnée avec son fœtus, elle devient indépendante des autres. Tout ce qu'elle ressent, c'est le plaisir d'être femme féconde apte à donner la vie, d'être enceinte habitée par un autre corps. C'est ce qu'a dit Dolto (1982, p. 57), affirmant que toute femme rêve de posséder un enfant. Celui-ci s'avère "le don de son père dont elle désire porter un fruit symbolique de féminité, et par lui rendue fertile." Bien sûr c'est l'état de Camille qui a du mal à cacher sa grossesse parce que sa "poitrine gonflée tente de révéler la vérité" $\left(U T P R^{3}, \mathrm{p} .69\right)$ et son ventre paraît "chaque jour plus rond que la veille." (UTPR, p.170)

Face à sa crise, Camille compte sur l'humour pour qu'elle puisse alléger ses tensions. Frappée de la décision de son compagnon, elle se moque de la cruauté de sa situation en disant: "Les deux barres ont perdu de leur enthousiasme et sont à présent légèrement rosées, comme timides ou honteuses." (UTPR, p. 13) Cet humour révèle l'inconscient parce qu'il naît d'une interaction et l'humoriste ne peut pas le mobiliser. La narratrice nous fait rire aux larmes lorsqu'elle annonce la fuite de son compagnon. Celui-ci s'en est allé en la laissant dans une situation difficile. Elle s'adresse à son fœtus monoparental et lui dit: "Il faudra que je sois ta mère et ton père à la fois, je me laisserai pousser la moustache pour que tu tombes amoureuse de moi et te répondre qu'on ne peut pas épouser son papa." (UTPR, p.159) Ceci lui permet de résister à l'anxiété, d'éviter la peur et être calme. La narratrice reprend l'humour jusqu'au bout. Imaginant qu'elle donne à sa fille, son premier bain, elle se demande qui va les prendre en photo. Elle dit: "Il faut que j'achète un retardateur, si tu veux tu pourras l'appeler papa." (UTPR, p.190)

Il nous serait important de relier les deux ouvrages de notre corpus à leur contexte, c'est-à-dire l'état des choses qui préparent à leur parution. Ce qui nous aide à bien saisir la double nature des deux romancières en tant que créatrices et femmes vivant dans une société et à révéler leur personnalité inconsciente ou leur mythe personnel qui

3 - UTPR est une abréviation d'Un tout petit rien. 
est à la base de la psychocritique. Le schéma de Mauron (1996, p. 330) détermine les composantes de ce mythe:

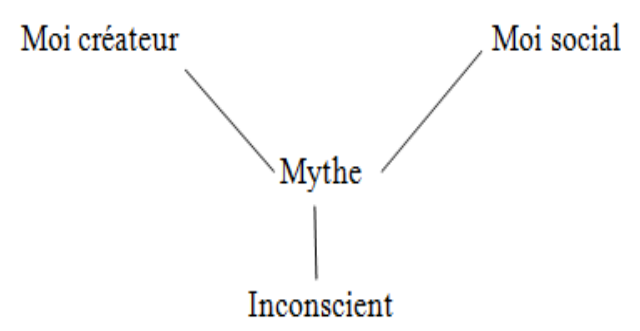

Disons que chaque créateur a son propre mythe personnel qui montre à sa façon ses processus psychiques. Celui-ci s'avère "le phantasme le plus fréquent chez un écrivain, ou mieux encore l'image qui résiste à la superposition de ses auvres." (Mauron, 1996, p. 300) Afin de mettre en relief le mythe personnel chez les deux romancières, nous allons indiquer les facteurs qui leur ont donné le désir d'écrire, les dimensions autobiographiques dans les deux romans de notre corpus, ainsi que leur deuil et leur expérience pendant la grossesse et l'accouchement.

\section{Le moi créateur dans Doniazade et Un tout petit rien}

Les deux ouvrages de notre corpus appartiennent au récit autobiographique qui a connu une grande réussite sous tous ses aspects. Dans ces récits, l'auteur et le narrateur s'identifient étroitement si bien qu'il est difficile de séparer l'un de l'autre. L'auteur s'annonce dès le début du récit et se présente comme un agent qui parle à la première personne. En vertu de ce "je" qui se cherche, on tient l'autobiographie pour une quête d'identité à travers la représentation d'un événement réel. L'auteur reconstruit sa vie en la racontant. Il essaye de tisser des liens entre les souvenirs qu'il a vécus au passé et d'en constituer une histoire ayant un sens et utile à ses lecteurs.

L'écriture de soi paraît sous plusieurs formes: la confession, le journal, les mémoires écrites par une personne importante, relatant 
Ayman Amin ELGHANDOUR

"les événements historiques auxquels il a pris part;" (Marcou, 2001, p. 19) les carnets basés "sur des expériences précises pour en tirer des préceptes." (Miraux, 1996, p. 14) Mais ce qui nous importe ici, ce sont les deux volets principaux: l'autobiographie et l'autofiction qui imprègnent les deux ouvrages de notre corpus.

Nous tenons l'autobiographie pour récit réel où l'auteur exprime la réalité telle qu'elle est, tout en offrant de vraies personnes et des événements qui ont eu lieu. Nous pouvons dire que tout récit de vie devient autobiographie en respectant le pacte de Philippe Lejeune (1996, p. 14): " Récit rétrospectif en prose qu'une personne réelle fait de sa propre existence, lorsqu'elle met l'accent sur sa vie individuelle, en particulier sur l'histoire de sa personnalité." En vertu de ce pacte considéré comme contrat de crédibilité entre le lecteur et son écrivain, celui-ci doit s'avouer sincèrement sans modifier la vérité; alors que l'autofiction s'avère un récit qui a partiellement un aspect fictionnel parce que son écrivain rédige la biographie d'une personne réelle et lui accorde des actions fictives. C'est Serge Dubrovsky (1993, p. 207) qui invente le terme autofiction en 1977 et le définit: "Fiction, d'événements et de faits strictement réels; si l'on veut, autofiction, d'avoir confié le langage d'une aventure à l'aventure du langage." Dans ces récits, l'auteur a principalement pour objectif de mêler le réel au fictif. Autrement dit, il crée un personnage fictif et lui attribue son identité réelle. Ainsi naît le pacte d'autofiction qui permet à celui qui raconte sa vie personnelle de la modifier relativement. En vertu de ce pacte, nous rencontrons une forme littéraire tout à fait différente de celle de l'autobiographie qui exige de ne pas citer le mot "roman" sur la couverture. Comme le romancier n'est pas obligé de dévoiler tout dans son ouvrage, Thierry Laurent nous informe qu'on peut saisir l'autofiction à travers d'autres détails cités par l'auteur dans ses interviews: "Les informations que donne l'écrivain pour passer son pacte d'autofiction avec le lecteur se trouvent dans le paratexte (titre, dédicace, épigraphe, préface, quatrième de couverture) et -le plus souvent- dans les entretiens qu'il accorde." (1997, p. 21)

Quant à May Telmissany, ses deux premiers romans mettent l'accent sur la société cairote, particulièrement Héliopolis, sa ville 
natale, créée par le baron Empain en 1905. La romancière égyptienne puise à son expérience personnelle, y racontant son enfance, sa jeunesse et sa maternité. Celle-ci est à la base de Doniazade dont l'héroïne, la narratrice et l'auteure ne sont qu'un seul individu. La narratrice nous raconte la perte de son enfant. Elle parle à la première personne, mais le "je" qui narre, est suffoqué. Elle confie la narration à son mari qui raconte le récit d'un autre angle. Les deux narrations sont typographiquement distinctes; celle du mari est écrite en lettres italiques. Cette technique polynarrative nous rappelle celle de Georges Perec dans $W$ ou le souvenir d'enfance. Ces deux narrations sont si complémentaires qu'elles présentent au lecteur tous les détails concernant la mort de l'enfant.

Bien que la narratrice ne porte pas de nom, elle ressemble à son auteure; les deux ont le même âge. Elle "a passé le cap de la trentaine," (Doniazade, p. 44) telle May Telmissany qui est née en 1965 et qui a publié Doniazade en 1997, deux ans après son écriture. Cela signifie que la romancière égyptienne avait 30 ans lors de la perte de son enfant. Autre chose fortifie leur ressemblance, chacune d'entre elles a deux frères. Le mari de la narratrice nous déclare qu'il a appelé les frères de sa femme. "Ils ont tardé. Le plus jeune est arrivé le premier." (Doniazade, p. 12) Rappelons ici que May a deux frères, il s'agit de Yasser et Khaled. Ce dernier qui est à la fois photographe et réalisateur, a trouvé sa fin le 4 septembre 2018, lors d'une collision au Sinaï du sud.

Après avoir perdu son enfant, la narratrice nous informe qu'elle a un autre, âgé de "quatre ans." (Doniazade, p. 49) Nous la voyons penser à "Chihabeddine qui rentrera bientôt avec son père." (Doniazade, p. 47) Son fils est amoureux de Salma, son amie de classe. La narratrice cite dans un autre endroit que "Chihabeddine était Gémeaux." (Doniazade, p. 51) De plus, le roman clôt avec la mère qui tombe enceinte, attendant l'arrivée de son deuxième enfant. Ces informations renforcent la ressemblance entre la narratrice anonyme qui raconte son histoire et May Telmissany, l'écrivaine du roman. En fait cette dernière a deux enfants: l'aîné Chihabeddine, né 
Ayman Amin ELGHANDOUR

en juin, c'est-à-dire jumeaux; il est spécialiste en anthropologie; le cadet $\mathrm{Ziad}^{4}$ qui a étudié le théâtre à l'Université de Ryerson. Remarquons que le nom de ce dernier est si proche de Zad, précédée par le mot arabe Donia qui signifie la vie.

Aussi la romancière et la narratrice ont-elles la même formation. Cette dernière parle bien le français, langue dans laquelle elle a lu "des ouvrages sur la grossesse et l'éducation des enfants," (Doniazade, p. 61) sans oublier Les aventures de Lucky Luke qu'elle avait lues à l'âge de douze ans. Elle se présente comme auteure; elle a écrit "Sculptures répétées" (Doniazade, p. 43) et a décidé à la fin du roman d'écrire "Doniazade." (Doniazade, p. 67) Ce sont les mêmes titres qu'avait présentés May Telmissany. Rappelons ici que la narratrice et Micky ou Mahy, l'héroïne de Héliopolis ont les mêmes traits. Nous la voyons auprès de son père dans la bibliothèque, regardant les illustrations dans "le Larousse rouge." (Telmissany, 2001/2003, p. 96) Dans une autre reprise, elle essaye d'écrire un récit "en le racontant au miroir." (Telmissany, p. 14) Si nous supprimons le "h" aspiré de Mahy, il nous restera May, le prénom de notre romancière.

Bien que le mari contribue partiellement à narrer l'histoire, la narratrice est consciemment prudente de ne pas le nommer. Elle dit: "Mon mari, je ne dis pas son nom." (Doniazade, p. 66) Elle nous informe qu'il parle le français. Ce dernier qui s'avère poète, dit: "J'ai pensé que j'écrirais pour elle [sa femme] un poème." (Doniazade, p. 20) En fait ce personnage romanesque n'est que le reflet du mari réel de notre romancière égyptienne; il s'agit du poète Walid El Khachab qui "est actuellement professeur des études arabes à l'Université de York, au Canada." (El Lackany, 2014) Il fait partie des poètes des années 1990. Il a écrit deux recueils de poèmes: Les morts ne consomment pas en 2000 et Celle qui est en 2013.

Il est évident que Doniazade est un texte autobiographique, regorgé de détails concernant la vie personnelle de May Telmissany.

${ }^{4}$-Ziad a commencé sa carrière d'acteur dans un feuilleton canadien, intitulé "Kim's convenience" (L'épicerie de Kim). 
Doniazade de May Telmissany et Un tout petit rien de Camille Anseaume

Nous y remarquons facilement les points communs entre la romancière et son héroïne. Celle-là a réussi à faire de sa vie un arrièrefond de ses écrits. C'est ce qu'affirme Wharton (2006, p. 104): "L'univers intérieur d'un artiste existe pour lui aussi fortement que le monde réel."

Quant à Camille Anseaume, elle a affirmé plus d'une fois qu'elle avait écrit un ouvrage autofictionnel. Dans son entretien avec le magazine Elle, la romancière dit: "En fait, c'est plutôt une autofiction. Je me suis inspirée d'une situation qui était la mienne, quand je suis tombée enceinte à 25 ans. [...] Mais j'ai tout de même modifié quelques éléments. A partir de ce moment-là ce n'est pas tout à fait moi." (Elle, 2014) Camille a créé un personnage fictif, l'héroïnenarratrice à qui elle a prêté son nom propre afin d'inventer une nouvelle existence et de garantir l'identité narrative. Elle a renoncé à l'autobiographie tout en choisissant le roman autofictionnel. Bref elle a adopté l'autofiction qui "est la forme romanesque dans laquelle se racontent les écrivains." (Vilain, 2005, p. 211)

Il est connu que les documents biographiques contribuent à éclairer toute œuvre romanesque et aident le critique à comparer le fictif au réel. En fait le manque des écrits concernant la vie personnelle de Camille Anseaume représente un obstacle à franchir. Cependant nous essayons au mieux d'examiner Un tout petit rien afin d'en dégager les points communs entre l'auteure et son héroïne.

Nous remarquons dès le début que le roman est dédié à Ninon, la fille de Camille Anseaume. La narratrice s'appelle Camille, femme moderne, vivant "au XXIe siècle, en France." (UTPR, p. 74) Elle nous informe dans un autre endroit qu'en "1989 [elle a] six ans. (UTPR, p. 44) Donc elle est née à la fin de 1983 ou au début de 1984. Agée de 25 ans, elle tombe enceinte. Neuf mois après sa grossesse, elle va au début du mois de Juin, "en salle d'accouchement." (UTPR, p. 236) De ce qui précède, on peut déduire qu'elle a accouché à la fin de 2009. Notre déduction est affirmée par Camille Anseaume en février 2016, 
lors de son interview avec le blog Serialmother. Elle annonce qu'elle a 32 ans et son "enfant Ninon, 6 ans et demi."

Bien que la narratrice habite à Paris, elle est d'origine rouennaise. Elle dit: "J'ai pris un billet pour Rouen, où vivent mes parents." (UTPR, p. 54) Elle nous a emmené à sa maison parentale que la romancière a reprise d'une manière détaillée dans Quatre murs et un toit. Elle est journaliste et internaute. Nous l'avons vu boucler "un

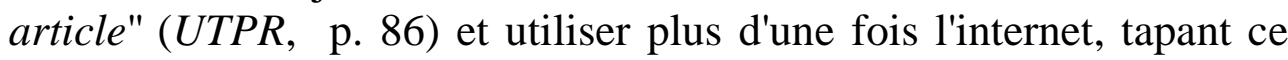
qu'elle veut savoir "sur un moteur de recherche." (UTPR, p. 188) Tout cela renforce l'identification de la narratrice et de Camille Anseaume qui est blogueuse, célèbre par son blog café de filles. Cette dernière exprime son amour pour Rouen, sa ville natale, elle dit: "C'est là que j'ai grandi. Je pense que les gens ont deux types de rapport avec leur ville d'origine: soit elle leur donne l'impression de régresser, soit il s'agit d'un projet de vie. Je fais partie du second groupe." (Solcito, 2014)

Camille s'est donc inspirée de sa grossesse imprévue et a écrit son roman semi sincère, comportant à la fois des détails vrais et d'autres fictifs. Face aux critiques qui considèrent son texte comme une autobiographie, elle affirme que "ceux qui [la] connaissent vraiment savent que ce n'est pas le cas." (Parole de mamans, 2014) Elle nous met consciemment à l' écart de son enfance, de ses parents. Aucun texte ne reflète sa vie personnelle, même sur internet. C'est ce qui nous empêche d'examiner la crédibilité de certains faits cités par la narratrice. A titre d'exemple, cette dernière nous raconte que lorsque son père était inapte à assumer la responsabilité de quatre enfants, a mis la plus petite en route; il murmure tristement qu'elle ne reviendra jamais parce qu' "elle est au ciel." (UTPR, p. 45) Nous nous demandons si cet événement est réel ou la romancière l'a inventé pour la structure dramatique de son ouvrage. En vertu de cet événement, la mère refuse jusqu' au bout la grossesse de sa fille et oblige tous les membres de la famille à adopter son opinion.

Les deux romancières égyptienne et française ont pu mettre la technique romanesque au service de leurs récits de vie. Chacune 
Doniazade de May Telmissany et Un tout petit rien de Camille Anseaume

d'entre elles a donné un titre expressif à son roman. D'une part, May Telmissany a choisi le nom de Doniazade qui nous rappelle ce personnage féminin, présent dans Mille et une nuits, témoin de la lutte de sa sœur Shéherazade face au roi injuste. Tout au fil du roman, l'auteure tente de susciter l'enfant tout en concentrant sur la vie de sa mère. D'après Bahaa Abd El Megid (2018, p.132): "La mort de l'enfant est le début d'une nouvelle étape dans laquelle la narratrice essaye de refléter plusieurs étapes de sa vie,"5 d'autre part, Camille Anseaume a choisi un titre énigmatique $U n$ tout petit rien qui nous pousse à nous demander s'il s'agit d'un fœtus si petit qu'il n'est rien. Cependant il a bouleversé la vie de la jeune narratrice, devenue mère célibataire. Nous l'écoutons dire: "J'étais pleine d'un vide au milieu duquel nageait l'infiniment petit." (UTPR, p. 12)

Comme les deux ouvrages de notre corpus appartiennent aux récits de vie, May Telmissany et Camille Anseaume ont parfaitement mis l'accent sur la mère narratrice, au détriment d'autres personnages secondaires, simples et marginaux. Exceptionnalisons le mari dans Doniazade, qui reste auprès de sa femme et à qui l'auteure égyptienne a confié une partie de la narration. Cet acte de brièveté et de concentration rend les deux textes proches de la nouvelle qui décrit un seul événement tout en évoquant des impressions personnelles. C'est ce qu'ont affirmé les deux romancières. L'une "a essentiellement écrit Doniazade, [son] premier roman, influencée par l'esprit de la nouvelle. [...] Les textes ont été si organisés dans un certain contexte qu'ils se rapprochent de l'univers romanesque." (Albyan, 1998, p. 5) De sa part, l'autre dit: "Tout est parti d'une nouvelle écrite pour le concours E-crire aufeminin.com. [...] La nouvelle commence à l'annonce de la grossesse et s'arrête à la prise de la décision. J'ai mis 9 mois (sic!) à écrire ce qu'il pouvait y avoir entre les deux." (Isabelle, 2014)

Dans les deux romans, l'auteure, l'héroïne et la narratrice sont identiques. Comme cette dernière est chargée de raconter son récit à

5ـ "موت الطفلة هو بداية مرحلة تحاول بها الساردة أن تعكس مر احل متعددة من حياتها" 
Ayman Amin ELGHANDOUR

un destinataire, nous observons l'absence du dialogue et l'existence de l'écriture plate et blanche où chaque ligne met en lumière le doute, les émotions, les joies et les peines. La sincérité des deux auteures nous met au cœur de leur intimité. S'ajoute à cela la légèreté qui distingue ce qu'on appelle le premier roman, ainsi que les traits de l'écriture moderne, marquée par les phrases éclatantes et les courts chapitres. En ce qui concerne Doniazade, Édouard Al-Kharrât (1990, p. 15) affirme que ce roman appartient à "la tendance moderniste qui marque à la fois une mutation d'une importance exceptionnelle de la sensibilité littéraire, et un défi de taille aux normes et aux conventions littéraires établies." Ceci paraît de même chez la romancière française, ayant l'habitude de raconter ses expériences à ses lecteurs à travers son blog. Elle dit: "Les petits chapitres de mon roman étaient écrits à la façon de billets de blog." (Parole de mamans, 2014)

Il est évident que l'auteure-narratrice parle à la première personne dans les deux romans étudiés. Elle nous raconte son histoire dans laquelle elle est l'héroïne, il s'agit de sa grossesse et son accouchement. La narratrice est à la fois autodiégétique et intradiégétique. Si la narratrice essaye dans Doniazade d'évoquer ce qui s'est passé, l'autre dans $U n$ tout petit rien, se rappelle tout en inventant le réel. Cette différence justifie la narration adoptée par les deux romancières. May Telmissany a compté sur la narration simultannée, "se déroulant théoriquement en un temps sensiblement identique à celui des événements." (Valette, 1993, p. 130) Elle annonce qu' "elle a écrit Doniazade, trois jours après la mort de l'enfant;" (Abouzeid, 2005) d'autre côté, la narration est ultérieure dans Un tout petit rien dont l'auteure s'est contentée d'écrire une nouvelle. Mais après avoir obtenu le prix E-crire aufeminin, elle a décidé d'écrire son roman et y raconter son expérience passée.

\section{L'inconscient des deux narratrices enceintes et leur conflit psychique}

La grossesse est étroitement liée avec l'écriture. Ancrée dans son corps féminin en transformation, l'auteure enceinte se consacre à enregistrer ce qu'elle ressent. Les changements physiques de l'enceinte 
Doniazade de May Telmissany et Un tout petit rien de Camille Anseaume

lui imposent des malaises troublants et des pensées anxieuses, résultant de son inconscient. C'est ce qui la pousse à communiquer ses craintes et ses doutes à ses lecteurs afin de recevoir leur soutien. D'ici, nous trouvons dans la littérature beaucoup de romans féminins ayant pour thème la grossesse, l'accouchement et l'avortement. A titre d'exemple, nous en citons Les armoires vides, L'événement d'Annie Ernaux et Instruments des ténèbres de Nancy Huston. Celle-ci nous affirme "qu'il n'y a pas d'incompatibilité intrinsèque entre écriture, érotisme et maternité." (Huston, 1990, p. 93)

L'originalité du corps féminin réside dans la grossesse et l'accouchement qui donnent à la femme sa spécificité. Bien que celleci reçoive la semence de l'homme, c'est son corps qui ressent et éprouve tous les cycles de la vie, dès la grossesse jusqu'à l'allaitement. Son ventre habité d'un foetus, lui fait éprouver des sensations et des changements tangibles lorsqu'elle écrit un ouvrage et y essaye de comprendre ce qu'elle a traversé. C'est ce qu'exprime Ali El Raie à propos de Doniazade: "Ce roman représente une expérience unique qui ne peut être écrite que par une femme car c'est la femme qui accouche et ressent la douleur de la perte." (Al Alamy, 2014)

May Telmissany et Camille Anseaume nous invitent à partager leur quotidien et à nous identifier à leur malaise. Elles nous présentent le drame des femmes lorsqu'elles tombent enceintes. Malgré le thème identique, l'action dramatique est différente dans les deux romans. Doniazade s'ouvre sur la scène de l'enfant morte "dans son petit linceul blanc" (Doniazade, p. 9) qui pousse la narratrice à faire retour en arrière pour raconter les détails de son histoire. Alors que la scène de l'accouchement clôt le roman français dont la narratrice s'est intéressée à mettre l'accent sur la grossesse, cette période bouleversante. C'est ce qui nous justifie pourquoi le corps est plus présent dans le roman français que dans celui de May Telmissany. Cette dernière paraît dès le début, blessée et sa blessure est à la fois physique et psychique. Malgré sa prudence d'aller "passer une échographie," (Doniazade, p. 16) elle a perdu son enfant. Bien plus elle ressent sa culpabilité de cette mort qu'elle subit comme un 
Ayman Amin ELGHANDOUR

fardeau. Elle dénie entièrement son corps féminin au point de dire: " $A$ présent mes seins pendent inutiles." (Doniazade, p. 21) Elle subit des conséquences psychologiques qui la rendent triste et la marquent jusqu'à la fin du roman.

Quant à Camille Anseaume, elle révèle d'une manière détaillée, le quotidien de la narratrice enceinte dont le corps change graduellement. Nous la voyons alterner deux pantalons de grossesse au lieu de son jean qu'elle avait du mal à fermer. Pour bien remarquer ses transformations, elle "passe des heures à filmer [son] ventre, à le prendre en photo." (UTPR, p.190) Plus le fotus grandit, plus la position debout lui paraît difficile, son "ventre encombré par son propre poids est tendu, comme prêt à exploser." (UTPR, p.189) Elle s'allonge afin d'éviter ce qui la dérange et libérer sa peau de la loi de la pesanteur. Elle nous raconte de même son allée chez l'obstétricien: " $A$ sa demande, j'étale sur mon ventre un liquide transparent et visqueux, il branche son écran et pose sur mon ventre luisant un objet froid qu'il fait glisser, révélant le son magique de ton cour." (UTPR, p.157) Tous ces petits détails expriment la spécificité de l'écriture féminine. Aucun homme ne peut traiter habilement ce thème féminin. La romancière enceinte est la seule qui puisse ancrer sa concentration dans le ventre puisqu'elle porte en elle deux vies: la sienne et celle de son fœtus.

Dès le début de Doniazade, May Telmissany présente à son lecteur un événement tragique où se côtoient la mort et la naissance. Elle nous conduit à la chambre 401 où la narratrice voit son enfant morte pour la première et la dernière fois. Dans cette scène, l'enfant fait ses adieux à sa mère qui lui donne un nom pour qu'on puisse rédiger un acte de décès. La narratrice exprime son deuil progressif parce qu'elle est à la fois témoin de cette scène tragique et responsable de la mort de son enfant en vertu du rapport médical qui "indiquait que la mort avait eu lieu dans l'utérus. Décollement du placenta." (Doniazade, p. 15)

Malgré sa mort, l'enfant reste vivant tout au long du roman, dans la mémoire de sa mère qui perd son équilibre psychique et devient hyper angoissée. Elle dit: "J'ai senti si souvent que nous étions 
Doniazade de May Telmissany et Un tout petit rien de Camille Anseaume

quatre personnes dans la famille!" (Doniazade, p. 21) Ce sentiment revient à la nature maternelle de la narratrice, contente d'être habitée d'une fille dont elle attend curieusement la venue, lui achetant "un joli berceau aux couleurs éclatantes, recouvert de tulle et de dentelles." (Doniazade, p. 13) Et plus sa grossesse se passe bien, plus l'enfantement s'approche et le temps de son attente diminue. Surprise par la perte de sa fille, née morte, elle refuse inconsciemment sa tragédie. Même si elle conçoit son absence, elle lui reste attachée au point qu'elle voit "son tombeau, tranquille, dans un coin de la chambre." (Doniazade, p. 25) Cette scène obsédante nous révèle à quel point se mêlent la conscience et l'inconscient chez la narratrice. Si l'une lui annonce la mort de la fille, l'autre la pousse à la conserver même si elle est un cadavre.

En fait, le sens du réel dévoile doucement à l'individu un univers hostile. Ce sens creuse le fossé entre lui et son milieu et influence passivement son équilibre psychique. Pour contrebalancer, il tend aux illusions et aux hallucinations tout en néant le réel. C'est ce qui nous explique pourquoi la narratrice essaye d'échapper au réel. A l'écoute du frottement des pieds nus, elle dit: "Quand j'ouvre les yeux je me retrouve, là, face à moi, toute petite, à peine trois ans. Menue, souriante comme le soleil. Je m'embrasse, m'étreins, m'appelle "Doniazade" - qui me ressemblait." (Doniazade, p. 25) Cette rencontre avec soi-même a permis à la narratrice d'évoquer l'image de l'enfant qu'elle était. Ce phénomène révèle indirectement les censures imposées par l'inconscient, visant à éveiller des souvenirs refoulés afin d'alléger la tension et renforcer les comportements de défense. Cette scène nous rappelle celle de Victor Segalen (1995, p.313) dans son Équipée où il cherche l'Alter égo. Il réussit à appeler le passé et rencontrer l'Autre qui "était [lui] de seize à vingt ans."

Comme nous l'avons déjà mentionné, May Telmissany a commencé à écrire Doniazade, trois jours après la mort de sa fille. Elle considère ainsi l'écriture comme un moyen thérapeutique. Il serait utile de rappeler ses dires: "A mes débuts, je disais en riant que l'écriture substitue le psychiatre." (Shérif, 2003) En fait l'écriture a 
Ayman Amin ELGHANDOUR

une double fonction dans le roman égyptien. D'une part, elle soulage la narratrice qui écrit "Doniazade, faisant appel aux lettres de son nom pour qu'elles [lui] donnent l'oubli;" (Doniazade, p.67) d'autre part, elle contribue à donner vie à la fille qui "n'a été rien de plus que ces quelques lignes." (Doniazade, p.21)

En écrivant, la narratrice a réussi à mettre son surplus à distance et le déposer sur le papier. Plus l'écriture soulage sa tension psychologique, plus elle surmonte ses angoisses et la mort, elle-même. Elle a décidé d'évoquer une nuit d'amour pareille à celle qu'elle avait déjà connue, "pour engendrer un nouvel enfant qui ne mourra pas." (Doniazade, p.44) Elle a essayé de modifier son milieu, annonçant sa séparation d'avec sa vie passée: sa démission, sa querelle avec un certain nombre de ses amies et enfin le changement de son médecin. Bien qu'elle tombe enceinte, elle exprime de nouveau sa tension en voyant le test de grossesse dont les deux barres en relief. Elle incite son nouveau médecin "à sauver le prochain enfant, comme s'il était irrémédiablement destiné à mourir." (Doniazade, p.60) Tout cela nous fait répéter les paroles de S. El Youssef (2001): " May Telmissany écrit essentiellement le conflit psychologique à multiples phases qu'elle subit pendant l'écriture."

Quant à Camille Anseaume, elle nous offre un récit poétique et affectueux qui met en lumière une double venue au monde: la naissance d'un enfant et celle d'une mère célibataire. Choquée de son conjoint qui est parti après avoir appris sa grossesse, celle-ci dit tristement: "Ça craint, j'ai appris il y a quelques heures que j'étais enceinte, mon amoureux pas amoureux s'est barré." (UTPR, p.29)

C'est le début de trois mois d'hésitation pendant lesquels la narratrice rencontre sans cesse ses amies, ses parents et son médecin. Le temps passe mais elle a du mal à prendre sa décision: se débarrasser de son fotus, reprenant naturellement sa vie ou le garder et l'élever toute seule? Nous la voyons dominée par la peur. Elle exprime son hésitation à la page 73 où il ya un tableau composé de deux colonnes: l'une présente les avantages et les risques de l'avortement que sa famille souhaite, alors que l'autre montre ceux de 
Doniazade de May Telmissany et Un tout petit rien de Camille Anseaume

la grossesse, soutenue par ses amies. C'est ce qui aggrave la situation et anime le conflit intérieur chez la narratrice qu'elle exprime d'une manière humoristique: "Aujourd'hui, quand on me demande: "Alors, tu vas faire quoi?" Je réponds que je ne sais pas et que je m'en fous. Je voudrais tirer à pile ou face." (UTPR, p.63)

La narratrice hésite ainsi entre sa maternité et sa liberté. Si elle choisit l'enfant, il sera sans père. C'est pourquoi, elle a décidé d'être auprès de son amant, prenant une pilule contraceptive au lendemain de leur relation. Cependant elle est tombée enceinte et celui-là l'a quittée toute seule, face à son tragique. Elle a pensé à l'avortement, ne dit-elle pas: "J'ai monté les sept étages en courant, et je les ai redescendus plus vite encore. Une fois, puis deux, puis trois." (UTPR, p.34) Épuisée, elle s'allonge sur son lit, demandant pardon à son fœtus. L'avortement lui paraît une culpabilité. Elle affirme catégoriquement: "J'ai décidé de garder ce bébé." (UTPR, p.94) Ce choix désigne l'inconscient chez la femme dont la grossesse et l'accouchement confirment sa féminité et sa maternité. Exceptionnalisons Annie Ernaux qui a choisi l'avortement pour se délivrer de son amant qui la méprisait. Persuadée que la grossesse est un emprisonnement imposé aux femmes, "l'étudiante dans l'événement semble faire un premier pas vers l'affranchissement quand elle décide d'avorter." (Nelson, 2004, p.78) C'est ce qui nous pousse à dire qu'Un tout Petit Rien est un cri contre l'avortement.

La grossesse permet à Camille de bien observer son corps en transformation et de s'adresser à son fœetus, lui disant: "Je voudrais juste que le monde comprenne que tu es une bonne nouvelle." (UTPR, p.145) Elle le présente ainsi comme si c'était le plus bel incident de sa vie. Les deux s'identifient au point que la narratrice utilise l'adjectif possessif "notre", exprimant la collectivité; elle dit" "Je me libère d'un secret. J'officialise notre union." (UTPR, p.115) Le texte fourmille aussi de plusieurs passages où la mère parle directement à son fotus, créant une relation vraiment étroite avec lui. Ses conversations sont présentées non seulement comme un moyen de communication, mais aussi comme une thérapie: la mère croit que la grossesse se passe bien 
lorsqu'elle parle à son fœtus; ses paroles sont si efficaces qu'elles contribuent à soulager ses doutes et ses peurs.

Aussi la narratrice exprime-t-elle son attachement à son fœtus à travers l'inconscient. Elle lui dit: "Quand je dors il semblerait que je ne rêve pas que de toi." (UTPR, p.120) Il est connu que les rêves reflètent les diverses transformations que subit l'enceinte. Ces rêves ont à la fois une influence sur son état physique et émotionnel parce que tous les événements qui s'y déroulent, l'enceinte les tient pour réels.

Après avoir appris qu'elle est enceinte, la narratrice commence à s'adapter à ses changements corporels, acceptant le fœtus vivant à l'intérieur de son ventre. Elle comprend aussi qu'elle doit modifier ses mauvaises habitudes; elle décide de ne pas fumer des cigarettes et renonce volontiers au vin afin de ne pas nuire à son fœtus "si petit, si fragile, suffoquant dans ce nuage de fumée et ces vapeurs d'alcools." (UTPR, p.43) De plus, nous la voyons prudente d'ouvrir le bouton de son pantalon, d'enlever son ventre et le ressortir au maximum pour que son fœtus ne se tourmente pas. Elle essaye d'être calme, cachant son inquiétude afin que son fœtus aille bien. Malgré sa fatigue après l'accouchement, la narratrice examine sa fille et lui dit: "On se retrouve toi et moi, j'en profite pour compter tes doigts. Tu en as dix. Ni plus ni moins." (UTPR, p.241)

Bien que la narratrice paraisse hésitée au début du roman, nous la trouvons à la fin "ancrée dans le présent et tendue vers un futur qui [la] fascine d'avance." (UTPR, p.220) Satisfaite de son choix, elle compare sa fille à une fleur dont la naissance annonce la venue du "printemps dans la salle d'accouchement." (UTPR, p.240)

En fait c'est la fille née qui donne à la fois naissance à la mère célibataire et à la jeune auteure. C'est elle qui lui offre la maternité et l'inspiration. Camille ne commence à écrire son roman que pour lui expliquer ce qui arrive tout en exprimant ses doutes, ses peurs et ses désirs. L'écriture lui est ainsi une thérapie. C'est ce qu'affirme la 
Doniazade de May Telmissany et Un tout petit rien de Camille Anseaume

romancière elle-même en disant: "L'écrit est vraiment le moyen de communication dans lequel je me sens le plus à l'aise." (Bréau, 2015)

Il est évident que l'inconscient, deuxième volet du mythe personnel, est à la base des deux ouvrages de notre corpus. C'est lui qui a poussé les deux romancières à écrire leurs récits de vie. Si l'une a connu son soulagement dans l'écriture, l'autre l'a utilisée pour faire de son foetus juge et témoin dans les conflits qu'elle a subis avec son conjoint. Aussi leurs écrits font-ils de cet enfant imaginaire, un autre plus réel et plus factuel. En écrivant, elles ont contribué à sécuriser leur présent et à assurer leur futur. Bien qu'elles mettent en relief leur grossesse et leur vie intime, elles ont de même révélé le social.

\section{Le moi social des deux auteures de notre corpus}

Mettons l'accent sur le moi social, la troisième composante du mythe personnel. Il va sans dire que tout récit de vie est essentiellement destiné à des lecteurs qui s'identifient à l'auteur. En lisant, chacun d'entre eux aperçoit sa propre histoire. Donc le récit de vie veut que le lecteur vive dans l'histoire de l'autre. Cela signifie que l'écriture intime s'adresse à la fois à l'individu et à la société parce qu'elle met en lumière l'état psychologique du protagoniste et le milieu qui l'entoure. Personne ne peut ignorer l'impact sociopolitique de ces récits dont l'auteur est divisé en deux: le moi créateur et le moi social. C'est ce qui nous explique pourquoi la littérature et la sociologie sont si étroitement liées qu'elles créent ce qu'on appelle la sociologie littéraire. Cette dernière qui "s'attache à restituer la vie d'une communauté ou d'un individu par le récit et la description, est souvent proche de la littérature elle-même." (Dortier, 1998, p.226) Nous pouvons dire que les deux auteures de notre étude font de la sociologie parce qu'elles présentent des faits sociaux en racontant leur vie personnelle.

May Telmissany et Camille Anseaume ont pu mettre l'accent sur leur grossesse et leur accouchement, deux thèmes chers à toutes les femmes. Les deux romancières sont ainsi passées de l'intime au 
Ayman Amin ELGHANDOUR

social, du personnel à ce qui touche les autres: le couple, l'amour, l'enfant attendu, le poids de la mère, les choix décisifs concernant la famille ... etc. Ceci a poussé les lectrices à compatir aux peines des deux héroïnes.

May Telmissany n'a cessé de jouer un rôle remarquable dans la société égyptienne, enseignant le français à l'université de Ménoufiya, le cinéma à l'Académie des Arts, sans oublier sa carrière de présentatrice à la Radio Le Caire. Elle a traduit son expérience à travers ses œuvres. Elle ne s'est pas contentée de montrer sa vie intime dans Doniazade, mais elle a mis en évidence des questions sociopolitiques. Aux dires de J. C. Ploquin (2014): "Ses romans reflètent un regard critique sur la société patriarcale égyptienne."

En tant que romancière et professeure universitaire, May Telmissany connaît bien la place considérable de la femme dans la civilisation pharaonique, devenue déesse telle Osiris. Il lui est naturel de se demander comment la société égyptienne a marginalisé la femme au $\mathrm{XX}^{\mathrm{e}}$ siècle. Dans cette société marquée par la polygamie, la femme se trouve prisonnière dans les harems, particulièrement pendant les années 50. Elle est considérée comme un objet aux mains de son mari. "Dès lors "la femme objet" n'a pas besoin de s'instruire, elle est là pour procurer le plaisir, pour être mère." (Tadié, 1996, p.49) A ce temps-là, il est honteux pour la plupart des familles, d'envoyer leurs filles aux écoles. Exceptionnalisons la classe bourgeoise dont les filles fréquentent les écoles françaises.

Bien que les mouvements féministes appellent à l'instruction des filles, c'est la Révolution de 1952 qui a rendu l'enseignement primaire obligatoire et l'a imposé aux enfants des deux sexes. Ceci montre à quel point l'État nassérien a soutenu les revendications du féminisme. Rappelons que "la constitution de 1956 accorde aux femmes le droit de vote." (Roussillon et Zryouil, 2006, p.53) Malgré cette réforme, les femmes souffrent encore, trouvées dans un système patriarcal où l'homme domine partout, exerçant son autorité absolue dans toutes les structures de l'État. Noual El Saadaoui (1991, p.167) observe que "le nombre élevé de femmes souffrant de névroses en 
Doniazade de May Telmissany et Un tout petit rien de Camille Anseaume

Egypte traduit les contradictions et les conflits qui divisent la société." Ce sont les femmes pauvres qui s'avèrent victimes, exposées aux fatigues et subissent des problèmes socio-économiques. On ajoute à cela d'autres angoisses concernant les femmes égyptiennes comme la peur du divorce et de la polygamie. En outre, la société ne se contente pas de dévaloriser la femme, mais elle l'accuse d'être le motif immédiat de l'harcèlement viril. Deux ans avant la parution de Doniazade, "en 1996, une jeune fille violée dans un bus que personne n'intervienne, se vit reprocher par le juge de n'avoir pas été voilée." (Zuhut, cité dans Roussillon et Zryouil, 2006, p.71)

Face à cette société où la femme est marginalisée, May Telmissany a choisi la gauche, décidant de parcourir le chemin de la résistance. Elle dit: "J'ai grandi ayant foi dans la gauche du cour, pas dans celle du parti, dans la révolution, pas au pouvoir."6 (Alkhaleej, 2015) D'ici la romanicière égyptienne a présenté son autobiographie pour exprimer à la fois son existence sociale et son acte révolutionnaire. Nous avons vu la narratrice dans Doniazade, exercer sa liberté, adressant sa critique à ses voisins chaotiques qui "faisaient creuser des conduits neufs pour l'eau potable et le drainage." (Doniazade, p.32) Après avoir pensé à appeler la police pour mettre fin à ce chaos, elle hésite et nous surprend en disant: "La police n'est pas au service de personne!" (Doniazade, p.33) Aussi évoque-t-elle Nasr Abou Zeid en marge de son récit. Le médecin lui dit: "Je vous ai vu à la dernière réunion qui s'est tenue pour Nasr Abou Zeid." (Doniazade, p.57) Cette évocation n'est pas gratuite. Ce dernier a écrit Critique de discours religieux, y présentant ses idées, appelant à une nouvelle lecture du Coran. Il a de même défendu la laïcité en Égypte. C'est pourquoi, les extrémistes musulmans l'ont obligé à l'exil en 1995 et un tribunal égyptien a confirmé un jugement en vertu duquel ce professeur universitaire devrait divorcer sa femme. Face à cette décision inique, le couple est parti pour Hollande.

6ـ "نشأت على الايمان بيسار القلب لا بسار الحزب, و بالثورة لا بالسلطة " 
Ayman Amin ELGHANDOUR

Influencée par son existence au Canada, May critique la société égyptienne et lui demande de vivre en paix et en harmonie. Elle dit que "dans d'autres pays du monde, il est possible de vivre dix fois mieux parce que des politiques y ont été faits pour favoriser la justice sociale, l'égalité, le respect de différences." (Deglise, 2017) Malgré son travail à l'extérieur, elle s'est engagée à la Révolution du 25 Janvier et a exprimé son désir de supprimer l'article 2 de la constitution égyptienne. Elle a "lancé une initiative en faveur de l'État civil ("dawla madaneya") en créant un site internet dédié." (Ploquin, 2014)

Quant à Camille Anseaume, elle a réussi à reprendre un chemin déjà parcouru par Nathalie Sarraute, Françoise Sagan, Marguerite Duras et Annie Ernaux. Celles-ci ont pu pénétrer le métier d'écrivain, quasi réservé au premier sexe. Elles ont déclaré leur présence, mettant un terme au silence des femmes. Grâce à leur résistance, la femme a graduellement occupé une place considérable dans les discussions intellectuelles et sa présence est devenue plus évidente sur la scène littéraire. Il serait utile de rappeler ici la maison d'édition "Des femmes," fondée en 1974, consacrée à publier seulement des ouvrages féminins.

Romancière et journaliste dans la presse féminine, Camille Anseaume est persuadée que l'écriture aide son auteur à surmonter les difficultés, face à un monde agressif. Avec Un Tout petit Rien, elle présente à ses lecteurs, son expérience et les émotions qu'elle a subies lors de la grossesse. Bien qu'elle exprime sa vie intime, elle s'adresse essentiellement aux futures mères qui poursuivent son blog. Elle publie son roman sur internet, souhaitant que celles-ci la soutiennent et admettent sa conduite. Cette intimité ne l'entrave pas d'aborder dans son ouvrage, d'autres thèmes, liés à sa grossesse. Elle met l'accent sur sa famille, ses amies et essaye d'enrichir sa propre histoire en ajoutant des détails amusants et surprenants. Tout cela rend l'ouvrage plus authentique. Ayant un double message, il exprime la crise personnelle de la narratrice et affirme la voix des femmes. La romancière y met l'accent sur un problème; il s'agit de la mère célibataire qui assume toute seule la responsabilité de son enfant alors que son partenaire 
Doniazade de May Telmissany et Un tout petit rien de Camille Anseaume

s'évade sans retour. Elle dit tristement: "Je pleure de rage, d'injustice de devoir supporter ça toute seule." (UTPR, p.172) Pour cela ses parents lui demandent de se débarrasser de ce fotus. Mais elle décide de le garder et par conséquent elle se trouve "seule contre tous." (UTPR, p.60) D'ici commencent ses débats intérieurs et ses conflits psychiques. Ces débats sont non seulement entre elle et sa conscience, mais aussi un cri contre une société injuste dont la pression pèse lourdement sur les femmes.

La narratrice remonte au code de la santé publique et en cite l'article L. 2212-1 en vertu duquel "la femme enceinte que son état place dans une situation de détresse peut demander à un médecin l'interruption de sa grossesse." (UTPR, p.35) Cette intertextualité montre à quel point la loi française donne à l'enceinte, la liberté du choix. Mais dans notre roman, l'hérö̈ne qui a décidé de garder l'enfant, assume toute seule la responsabilité de son fotus. C'est un cri adressé contre la société, ayant pour but d'obliger l'homme à aider financièrement son ex-épouse afin qu'elle puisse former et éduquer son enfant.

Il est évident que May Telmissany et Camille Anseaume ont présenté non seulement leur moi créateur et individuel, mais aussi leur moi social qu'elles ont acquis tout au long de leur vie. Ce dernier éveille en elles leur solidarité sociale et les pousse à exprimer leur expérience et leur vie intime en vue d'éclairer les autres. Les deux ouvrages ont révélé la complémentarité entre le moi individuel et le moi social au point qu'on ne peut pas séparer l'un de l'autre. Ce qui a créé un équilibre entre la liberté créative et les contraintes sociales, tout en révélant l'inconscient que les deux romancières ont présenté sous des structures bien choisies.

A la fin de notre étude, nous affirmons que May Telmissany et Camille Anseaume ont évoqué leur vie intime et y ont emprunté leur premier roman; l'une a mis l'accent sur son accouchement et la perte de son enfant, alors que l'autre, mère célibataire, a exprimé d'une manière détaillée ses émotions et son conflit psychique pendant sa 
Ayman Amin ELGHANDOUR

grossesse. Pour cela nous avons adopté la psychocritique pour mettre en évidence le mythe personnel des deux auteures tout en révélant leur moi créateur, leur moi social et leur inconscient. Cette méthode nous a aidé à interpréter les deux ouvrages et à déterminer les motifs de l'écriture. L'une a écrit pour donner vie à sa fille morte, présentant une sincère autobiographie, alors que l'autre a écrit une autofiction pour expliquer à sa fille, son hésitation et son oscillation entre la grossesse et l'avortement. L'écriture a joué un rôle thérapeutique pour les deux narratrices et les a aidées à mettre fin à leurs deuils,

Les deux romans qui sont découpés en pages courtes, se distinguent par la précision et l'écriture blanche pleine d'émotions. Nous y avons remarqué toutes les caractéristiques de la nouvelle: texte court, basé sur une seule histoire, mettant l'accent sur un petit nombre de personnages et dont l'intrigue compte essentiellement sur l'évolution psychologique du protagoniste. Les deux romancières ont écrit leurs ouvrages en s'intéressant à la narration sans rapporter la parole des personnages. Ceci revient à l'écriture de May Telmissany qui a commencé sa carrière comme nouvelliste. Aussi Camille a-t-elle écrit une nouvelle, évoluée plus tard pour devenir roman.

Les deux romancières prouvent que l'écrivain n'est pas créé pour être prisonnier dans une tour d'ivoire. Elles ont raconté leur grossesse et leur maternité pour éclairer d'autres femmes. Chacune d'entre elles a adressé une critique contre sa société; l'une a fait allusion au fanatisme religieux en Egypte, et l'autre a dénoncé la domination masculine, mettant l'accent sur la souffrance de mère célibataire. Hors de leurs écrits, May a soutenu la Révolution du 25 janvier, appelant à l'État civil, basé sur la citoyenneté; de sa part, Camille en tant que journaliste dans la presse féminine, a guidé ses lectrices, leur présentant son expérience. Mais ce qui reste à jamais, c'est leur autobiographie qui les immortalise et renforce la présence féminine, apte à la création et à la procréation. 


\section{Bibliographie}

\section{1- Corpus étudié:}

- Anseaume, C. (2014). Un tout petit rien. Paris: Éditions Kero.

- Telmissany, M. (2000). Doniazade (traduit en français par Mona Latif Ghattas ). Paris: Actes Sud.

\section{2-Autres ouvrages de Camille Anseaume:}

- (2016). Ta façon d'être au monde. Paris: Kero.

- (2018). Quatre murs et un toit. Paris: Calmann-Lévy.

\section{3-Autres ouvrages de May Telmissany:}

- (2014). A cappella (traduit en français par Richard Jacquemond). Paris: Actes Sud.

- (2003). Héliopolis (traduit en français par Mona Latif Ghattas). Paris: Actes Sud.

\section{4- Ouvrages généraux:}

- Al-Kharrât, E. (1990). Aperçu sur la création romanesque égyptienne moderne. Dans Rencontres autour de la littérature romanesque égyptienne traduite en français. Le Caire: Maison Galaxie pour l'édition et l'imprimerie.

- Boyer, A. - M. (1996). Éléments de Littérature comparée, III. Formes et genres. Paris: Hachette.

- Dolto, F. (1982). Sexualité féminine. Paris: Scarabée.

- Dortier, J. - F. (1998). Les sciences humaines: panorama des connaissances. Auxerre: Éditions Siences humaines.

- Doubrovsky, S. (1993). Textes en main. Dans RITM: Autofiction et Cie, 6. Nanterre: Centre des Recherches interdisciplinaire sur les textes modernes, Université Paris X.

- El Saadaoui, N. (1991) Femmes égyptiennes, tradition et modernité, (traduit de l'arabe par Essia Trabesi). Paris: Des femmes.

- Hassan, K. J. (2006). Le roman arabe (1834 - 2004). Paris: Actes Sud.

- Huston, N. (1990). Journal de la création. Arles: Babel.

- Laurent, T. (1997). L'œuvre de Patrick Modiano: une autofiction. Lyon: Presses Universitaires.

- Lejeune, P. (1996). Le Pacte autobiographique. (colln. Points). Paris: Éditions du Seuil. 
Ayman Amin ELGHANDOUR

- Marcou, L. (2001). L'autobiographie, anthologie. (Colln. Étonnants classiques). Paris: Flammarion,

- Mauron, C. (1996). Des métaphores obsédantes au mythe personnel, Introduction à la psychocritique. Tunis: Cérès Éditions.

- Miraux, J. P. (1996). L'autobiographie, écriture et sincérité. Paris: Nathan.

- Noël, J. - B. (1996). La psychanalyse du texte littéraire, Introduction aux lectures critiques inspirées de Freud. Paris: Nathan.

-Pichois, C. et Rousseau, A. - M. (1967). La littérature comparée. Paris: Armand Colin.

- Roussillon, A. et Zryouil, F. - Z. (2006). Être femme en Égypte, au Maroc et en Jordanie. Montreuil: Éditions Aux lieux d'être.

- Segalen, V. (1995). Équipée. Dans Euvres complètes (colln. Bouquins), 2. Paris: Robert Laffont.

-Souiller, D. (1997). Littérature comparée. Paris: Presses universitaires de France,

-Valette, B. (1993). Esthétique du roman moderne. Paris: Nathan.

-Vilain, P. (2005). Défense de narcisse. Paris: Grasset et Fasquelle.

-Wharton, E. (2006). Les règles de la fiction. Paris: Éditions Viviane Hamy,

\section{5- Articles et périodiques:}

- Abd El Megid, B. (2018, juillet-août). Oeil magique de May Telmissany: le lieu tel un texte ouvert. Dans Ibdaa (Création), 4. Le Caire: Organisme général égyptien du livre. P. 132 - 135.

- Nelson, J. - A. (2004, printemps). L'avortement chez Annie Ernaux. Dans Dalhousie French Studies, (69). Halifax: Université de Dalhousie. P. 73 - 81. Repéré à http://www.jstor.org/stable/40837579. -Tadié, A. (1996, octobre - décembre). L'univers des romancières égyptiennes: thèmes et formes dans la littérature féminine. Dans Peuples méditerranéens, (77). P. 47 - 94.

\section{6- Pages Web / Documents sur Internet:}

- Adnani, H. (2014). May Telmissany digne représentante d'une littérature égyptienne résolument moderne. Repéré le 15 janvier 2017 à http://adnani.over-blog.com/2014/04/may-telmissany-dignerepresentante-d-une-litterature-egyptienne-resolument-moderne.html - Al Bayan. (1998, 5 juin). Repéré le 20 novembre 2017 à http://www.albayan.ae/five-senses/1998-05-06-1.1009571 
Doniazade de May Telmissany et Un tout petit rien de Camille Anseaume

- Al Khaleej. (2015, le 10 août). Repéré le 15 novembre 2017 à http://www.alkhaleej.ae/supplements/page/d293714c-44f1-4dfe-a56b$4 \mathrm{dc} 78 \mathrm{ca} 29 \mathrm{f} 70$

- Al Alamy, A. (2014, 13 avril). Lecture dans Doniazade de May

Telmissany. Dans Al Watan. Repéré le 3 février à

http://alwatan.com/details/12991

- Al Youssef, S. (2001, 23 février). La captivité du deuil. Dans Al Shark Al Awsat, (8123). Repéré le 30 janvier 2017 à http://archive. aawsat.com/details.asp?issueno $=8070 \&$ article $=27471 \#$.WbnHkdKCzIU

- Babelio. (S. D.). Camille Anseaume. Repéré le 15 septembre 2018 à https://www.babelio.com/auteur/Camille-Anseaume/276309

- Bréau, A. (2015, 16 février). Le grand dilemme de Camille Anseaume dans "Un tout petit rien. Repéré le 10 juillet 2018 à http://www.terrafemina.com/vie-privee/famille/articles/53955-

grossesse-imprevue-le-grand-dilemme-de-camille-anseaume-dans-untout-petit-rien.html

- Deglise, F. (2017, 19 août). La pensée nomade de May Telmissany. Repéré le 10 novembre 2017 à http://www.ledevoir.com/culture/livres 1505946/entrevue-la-pensee-nomade-de-may-telmissany

- El Lackany, S. (2014, 22 janvier). Walid El Khachab: celle qui est. Dans Al Ahram Hebdo. Repéré le 23 mars à http://hebdo.ahram.org.eg/ NewsContent/1009/5/35/4918/WalidElKhachab-Celle-qui-est.aspx

- Elle.fr. (S. D.) Elle a fait un bébé toute seule, le choix de Camille Anseaume. Repéré le 20 novembre 2017 à http://www.elle.fr/LoveSexe/Mon-mec-et-moi/Articles/Elle-a-fait-un-bebe-toute-seule-lechoix-de-Camille-Anseaume-2693910

- Isabelle. (2014, 25 mars) Camille Anseaume: l'écriture est une matière qui glisse entre les doigts. Repéré le 10 janvier 2017 à http://www. monblogdemaman.com/camille-anseaume-un-tout-petitrien/

- Parole de mamans. (2014, 20 mai). Camille, du blog Café de Filles. Repéré le 17 janvier 2018 à https://paroledemamans.com/ma-vie-demaman/parole-de-blogueuses/camille-du-blog-cafe-de-filles-2

- Ploquin, J. C. (2014, le 4 mai). Un regard d'espérance sur l'Egypte malgré la répression. Repéré le 5 juin 2017 à http://paris- 
international. blogs.la-croix.com/un-regard-desperance-sur-legyptemalgre-la-repression/2014/05/04/

- Serialmother. (2014, le 8 février). L'interview de la serialmother, Camille Anseaume, écrivain. Repéré le 20 décembre 2017 à http://serialmother.yoopies.fr/linterview-de-la-serialmother-camilleanseaume-ecrivain/

- Shérif, S. (2003). May Telmissany: l'écriture est le seul espace possible où je pratique ma liberté, la démence et l'indiscrétion. Repéré le 6 avril 2018 à http://archive.aawsat.com/details.asp?section=19\&article= 196360\&issueno=9078\#.WbnGCtKCzIU

- Solcito.fr. (2014, février). L'interview Voyage de Camille Anseaume, écrivain. Repéré le 16 novembre 2017 à http://www.solcito.fr /2014/02/ linterview-voyage-de-camilleanseaume/

- Telmissany, M. (S. D.) Dans wikipedia. Repéré le 15 janvier 2018 à https://fr.wikipedia.org/wiki/May_Telmissany 